\title{
Fatores Genéticos e de Meio na Produção de Leite de Caprinos Leiteiros ${ }^{1}$ \\ Heraldo Cesar Gonçalves ${ }^{2}$, Martinho de Almeida e Silva ${ }^{3}$, Francisco Stefano Wechsler², Alcides Amorim Ramos ${ }^{2}$
}

\begin{abstract}
RESUMO - Objetivou-se, no presente estudo, avaliar a produção de leite de caprinos leiteiros da região sudeste do Brasil, com intuito de verificar os fatores de meio e estimar os parâmetros genéticos pelo método dos mínimos quadrados (MMQ). Os controles de 1336 lactações foram inicialmente ajustados pela função multifásica (difásica) e calculou-se a produção de leite total (PLT). Os dados foram provenientes de sete propriedades e três raças (Parda Alpina, Saanen e Toggenburg). A média e o erro-padrão da PLT estimados pelo MMQ foram de $635,31 \pm 39,75 \mathrm{~kg}$. A interação ano x estação do parto influenciou a PLT. Em um dos anos estudados, a PLT foi menor para as cabras paridas no final da estação. Nas três estações de parto, observou-se comportamento quadrático da PLT, em função dos anos de parto. Para as três estações, a PLT aumentou de 1986 até meados de 1990, decrescendo em seguida. A idade de máxima PLT foi observada aos 46,65 meses. Das três raças estudadas, observou-se que as raças Parda Alpina e Saanen apresentaram alternância de superioridade na PLT em algumas fazendas, porém maiores que a Toggenburg. Os coeficientes de herdabilidade e repetibilidade da PLT estimados pelo MMQ foram de 0,296 $\pm 0,079$ e 0,277 $\pm 0,033$, respectivamente. Estes resultados revelam baixa confiabilidade em poucas observações dessas características ou na inconsistência das estimativas da função multifásica. Como a PLT é uma característica limitada ao sexo, sugere-se o teste de progênie como método de seleção mais eficiente para os reprodutores e uso de inseminação artificial como processo de disseminação do material genético selecionado.
\end{abstract}

Palavras-chave: caprinos, herdabilidade, produção de leite, repetibilidade

\section{Genetic and Environmental Factors Affecting Milk Production from Dairy Goats}

ABSTRACT - The objectives of this study were to evaluate the influence of environmental factors on milk production from dairy goats raised in the Brazilian Southeast and to estimate genetic parameters for this trait through least squares. The data originated from seven farms and three breeds (Alpine Brown, Saanen and Toggenburg). Milk yields obtained from 1,336 lactations were initially fitted to a diphasic function, whereby total milk production (TMP) was estimated. The overall mean and standard error for TMP were $635.31 \pm 39.75 \mathrm{~kg}$. A significant year $\mathrm{x}$ kidding season interaction was observed. In all three kidding seasons a quadratic yearly trend of PLT was observed: it increased from 1986 until mid-1990, decreasing thereafter. Age of maximum PLT was estimated as 46.65 months. Alpine Brown was superior to Saanen in some farms, the opposite occurring in others, but both showed higher PLT values than Toggenburg. Estimated heritability and repeatability coefficients were $0.296 \pm 0.079$ and $0.227 \pm 0.033$, respectively. These results show low reliability due to the small number of observations or to inconsistent PLT estimates obtained through the biphasic function. Since PLT is a sex-limited trait, it is suggested the use of progeny tests as the most efficient selection method for bucks and artificial insemination for spreading the selected genetic material.

Key Words: goat, heritability, milk production, repeatability

\section{Introdução}

Nos últimos anos, a caprinocultura no Brasil vem se consolidando como importante alternativa pecuária, principalmente para o pequeno produtor, que emprega mão-de-obra familiar. Entre os fatores que têm colaborado para essa consolidação, destacam-se o preço atrativo do leite, pelo produtor e consumidor, e as características particulares do leite, consumido por pessoas que possuem intolerância ao leite bovino.
Nas regiões sul e sudeste do Brasil, predominam as raças caprinas exóticas de origem européia: Saanen, Parda Alpina, Toggenburg e Anglo Nubiana, especializadas para a produção de leite, e animais oriundos de cruzamento absorvente em direção a estas raças exóticas.

Embora presente há algumas décadas, pouco se conhece sobre o desempenho dessas raças e são escassas as estimativas de parâmetros genéticos das características produtivas no Brasil, com um número consistente de observações.

\footnotetext{
1 Parte da tese apresentada à UFV pelo primeiro autor, para obtenção do título de "Doctor Scientiae". Financiada pela FAPEMIG.

2 Professor da FMVZ - UNESP Campus de Botucatu, 18618-000 - Botucatu, SP. E.mail: heraldo@fca.unesp.br

3 Professor da UFMG - Bel Horizonte - MG.
} 
Efeito de ano na produção de leite tem sido observado em raças nativas tropicais e subtropicais em seus países de origem (SINGH et al., 1970; PRAKASH et al., 1971; BARHAT e CHOWDHARY, 1978; MAVROGENIS et al., 1984), em cabras especializadas nos Estados Unidos (ALDERSON e POLLAK, 1980) e em cabras nativas, exóticas e seus mestiços, num total de 16 grupos genéticos no México (MONTALDO et al., 1981).

$\mathrm{O}$ efeito da estação ou época do parto sobre a produção de leite pode ser explicado, em grande parte, pelas alterações climáticas, as quais podem ter efeito direto ou indireto sobre os animais. Os efeitos diretos são os que alteram o comportamento dos animais. Segundo QUITTET (1978), o consumo de alimentos em dias de chuva ou de forte calor pode chegar a ser nulo. APPLEMAN e DELOUCHE (1958) relataram que as cabras apresentam o máximo consumo entre 0 e $10^{\circ} \mathrm{C}$, diminuindo paulatinamente à medida que a temperatura aumentou até $40^{\circ} \mathrm{C}$. Os efeitos indiretos estariam associados às ofertas qualitativa e quantitativa dos alimentos volumosos oferecidos aos animais nas diferentes épocas.

Efeito significativo de estação na produção de leite de cabras da raça Beetal foi reportado na Índia por (PRAKASH et al., 1971; SINGH et al., 1970). Para cinco raças nos Estados Unidos, ALDERSON e POLLAK (1980) observaram efeito de estação e interação de idade x estação, ambos significativos. As cabras paridas dentro da estação de parto e com idade superior a 40 meses apresentaram maiores médias de produção de leite.

No México, MONTALDO et al. (1981) relataram que as cabras de raças exóticas e suas mestiças com cabras crioulas que pariram no início da estação (janeiro e fevereiro) produziram mais leite que aquelas que pariram no final da estação (março-abril), e estas mais que as paridas fora da estação (maio-dezembro).

Nos Estados Unidos, GIPSON e GROSSMAN (1989) utilizaram a função bifásica para ajustar a curva de lactação de cinco raças de cabras (Parda Alpina, La Mancha, Anglo Nubiana, Saanen e Toggenburg) e observaram efeito de estação (inicial e final) na produção de leite aos 305 dias de lactação em ambas as fases. A produção de leite aos 305 dias na primeira fase, da função difásica, foi maior para as lactações iniciadas no final da estação $(263,6 \mathrm{~kg})$ que as de início de estação $(95,1 \mathrm{~kg})$. Para a segunda fase, a situação se inverteu, em que as lactações iniciadas no princípio da estação apresentaram maior produção de leite $(764,6 \mathrm{~kg})$ que as iniciadas no final $(616,9 \mathrm{~kg})$.
Em Chipre, nas cabras nativas da raça Damascus (MAVROGENIS et al., 1984), e no Egito, em cabras Alpinas Francesas, MOURAD (1992) verificaram que as cabras paridas na primavera apresentaram menores produções de leite que nas outras estações.

$\mathrm{Na}$ Espanha, em cabras crioulas da ilha de Tenerife, FRESNO et al. (1991), e na Noruega, STEINE (1975), também observaram efeito significativo de estação na produção de leite.

Por outro lado, BARHAT e CHOWDHARY (1978), na Índia, não observaram efeito de estação na produção de leite de cabras Rajasthani.

De acordo com GALL (1981), é difícil separar os efeitos de idade e de peso do animal sobre a produção de leite. Como não é prática rotineira avaliar o peso dos animais nas criações comerciais, esses dados não estão disponíveis; assim, a maioria dos trabalhos relata o efeito da idade sobre a produção de leite.

Em geral, as cabras de primeiro parto, em virtude do incompleto desenvolvimento corporal e fisiológico, produzem menos leite que as adultas.

A maioria dos trabalhos revisados indica que a idade de máxima produção está acima de três e abaixo de 7 anos (RONNINGEN, 1965; STEINE, 1975; PRAKASH et al., 1971; CHAWLA e BHATNAGAR, 1984). Já SINGH e SINGH (1974) relataram que a máxima produção ocorreu na segunda lactação na raça Jamnapari.

Além desses efeitos citados, ALDERSONe POLLAK (1980) encontraram efeito significativo da interação idade x estação de parto e MONTALDO et al. (1981) de ordem de parto x época sobre a produção de leite.

Usando uma função difásica para o ajustamento da curva de lactação de cabras e dividindo a produção total (305 dias), em duas fases, GIPSON e GROSSMAN (1989) não encontraram efeito de ordem de parição na produção de leite em nenhuma das fases. Já FRESNO et al. (1991) determinaram que a ordem do parto foi responsável por $0,9 \%$ da variação total da produção de leite, a qual aumentou da primeira até a quarta lactação.

Estudando 550 lactações de 110 cabras Alpinas Francesas, MOURAD (1992) observou efeito significativo de ordem de parto na produção de leite, que aumentou até a terceira parição e permaneceu constante até a quinta.

Por outro lado, BARHAT e CHOWDHARY (1978) não encontraram influência significativa de ordem de parto sobre a produção de leite de cabras da raça Rajasthani na Índia.

Existe grande diversidade na produção de leite de 
Rev. bras. zootec.

raças caprinas. As raças européias, de maneira geral, são especializadas para produção de leite, externando todo seu potencial quando exploradas em regiões de clima temperado. Por outro lado, quando em regiões tropicais, apresentam desempenho inferior, porém ainda assim superior ao das raças nativas de regiões tropicais e algumas vezes superior aos dos mestiços (BARHAT e CHOWDHARY, 1978; CHAWLA e BHATNAGAR, 1984; MONTALDO et. al., 1981; e KANUJIA et al., 1988).

Segundo GILL e DEV (1972), os desempenhos de duas raças exóticas (Parda Alpina e Anglo Nubiana) na Índia, mantidas em estabulação, foram de 310,59 e $289,46 \mathrm{~kg}$ de leite por lactação, médias estas superiores às das raças nativas lá exploradas.

No Brasil, para um rebanho específico da raça Saanen, foi constatada a média de $572,57 \mathrm{~kg}$ (RIBEIRO, 1997).

Nos Estados Unidos, ALDERSON e POLLAK (1980) relataram que a raça foi significativamente responsável por 29,2\% da variação total nas produções de leite, que apresentaram valores de 977, 960, 935, 925 e $769 \mathrm{~kg}$ para as raças Saanen, Parda Alpina, Toggenburg, LaMancha e Nubiana, respectivamente.

Usando um modelo multifásico, que divide a curva de lactação em duas fases, GIPSON e GROSSMAN (1989) relataram que as produções de leite (305 dias) para a primeira e segunda fases foram similares para as cinco raças, sendo para a primeira parição e primeira fase 197,$52 ; 148,25 ; 168,13 ; 201,23$; e $189,13 \mathrm{~kg}$ e para a primeira parição e segunda fase 675,$28 ; 604,95 ; 536,5 ; 698,67$; e 679,2 kg para as raças Parda Alpina, LaMancha, Nubiana, Saanen e Toggenburg, respectivamente.

Na França, BOICHARD et al. (1989) ressaltaram que a superioridade da raça Saanen $(54,7 \mathrm{~kg}) \mathrm{em}$ relação à Parda Alpina para a produção de leite da primeira lactação foi em razão da maior duração da lactação (9,5 dias) e do peso corporal da Saanen. Esses autores observaram também que a média de produção foi inferior nos rebanhos com as duas raças, sendo a redução maior na raça Saanen $(-41 \mathrm{~kg})$ que na Parda Alpina (-26 kg).

\section{Herdabilidade}

A herdabilidade é extremamente importante nos programas de melhoramento genético, pois quantifica a porção da variância fenotípica total causada pela variação dos valores genéticos aditivos. Dessa forma, as estimativas de herdabilidade permitem a pre- dição dos ganhos genéticos esperados e dos valores genéticos dos indivíduos de uma população.

No Brasil, estimativas de herdabilidade para produção de leite e outras características de caprinos, com número expressivo de observações, são escassas. Para um rebanho específico da raça Saanen, a herdabilidade foi estimada em 0,09 (RIBEIRO, 1997).

A literatura cita valores de herdabilidade para a produção de leite entre 0,16 e 0,72 (RONNINGEN, 1965 e 1967; STEINE, 1976; BOUILLON e RICORDEAU, 1976; ILOEJE et al., 1981; KENNEDY et al., 1982; SULLIVAN et al., 1986; SINGH et al., 1970; PRAKASH et al., 1971; MAVROGENIS et al., 1984; CONSTANTINOU et al., 1985).

\section{Repetibilidade}

As estimativas de repetibilidade para produção de leite são também escassas na literatura.

O trabalho mais antigo revisado (KUMAR et al., 1962) relata repetibilidade de 0,18 $\pm 0,06$ para a produção de leite na raça Beetal, na Índia.

RONNINGEN (1965) relatou repetibilidade para produção de leite de 0,40 em 4571 cabras estudadas na Noruega, e RONNINGEN (1967), de 0,62 e 0,63 para análises que incluíram rebanhos com tamanho inferior e superior a 10 cabras, respectivamente. Resultado semelhante de 0,57 foi também obtido na Noruega por STEINE (1976).

Utilizando cabras Parda Alpina, Saanen e Toggenburg, KENNEDY et al. (1981), nos Estados Unidos, estimaram repetibilidades para produção de leite de 0,45 e 0,48 para as regiões Norte e Nordeste, respectivamente.

As repetibilidades para produção de leite estimadas por GROSSMAN et al. (1986), nos Estados Unidos, foram 0,$42 ; 0,38 ; 0,45 ; 0,44$; e 0,41 para as raças Parda Alpina, LaMancha, Anglo Nubiana, Saanen e Toggenburg, respectivamente.

A análise conjunta envolvendo as raças Parda Alpina, Granadina, Anglo Nubiana, Saanen e Toggenburg, realizada por MONTALDO et al. (1982), no México, revelou estimativas de 0,59 para a produção total e de 0,73 para a produção diária de leite.

Menor valor foi recentemente estimado para a raça Saanen no Brasil, 0,20 (RIBEIRO, 1997).

Foi objetivo do presente trabalho avaliar os fatores de meio que influenciam a produção de leite de caprinos no Brasil e estimar os parâmetros genéticos pelo método dos mínimos quadrados (MMQ). 


\section{Material e Métodos}

Foram utilizadas 1336 observações de 678 cabras filhas de 95 bodes de três raças (1 - Parda Alpina, 2 - Saanen e 3 - Toggenburg), de sete fazendas, no período de 1986 a 1992, sendo que os reprodutores utilizados tinham pelo menos duas filhas. Os valores observados para a produção de leite total (PLT) apresentaram média de $576,89 \mathrm{~kg}$, variando entre 72,66 e $2305,40 \mathrm{~kg}$.

Os dados de controle de leiteiro contidos nas fichas zootécnicas registrados pelos criadores e do controle oficial realizado pela Associação Brasileira de Criadores foram inicialmente ajustados individualmente de acordo com a função difásica, com o intuito de estimar a produção de leite total na lactação.

A função multifásica foi primeiramente utilizada para descrever a curva de crescimento de frangos de corte de ambos os sexos (GROSSMAN e KOOPS, 1988a). Posteriormente, GROSSMAN e KOOPS (1988) utilizaram-na para descrever a curva de lactação das vacas

GIPSON e GROSSMAN (1989) foram os primeiros a ajustar a curva de lactação de caprinos por meio da função difásica, definida como:

$$
Y_{t}=\sum_{i=1}^{2}\left\{a_{i} b_{i}\left[1-\tanh ^{2}\left(b_{i}\left(t-c_{i}\right)\right]\right\}\right.
$$

em que $Y_{t}=$ produção de leite em $(\mathrm{kg})$, ao tempo $t$ (dias de lactação); tanh = tangente hiperbólica; $\mathrm{a}_{\mathrm{i}}, \mathrm{b}_{\mathrm{i}}$ e $c_{i}=$ parâmetros estimados pela regressão nãolinear; $\mathrm{i}=1$ e 2 e refere-se à fase.

Para estimar os parâmetros da função difásica, foi usado o procedimento REGREGN do programa SAEG (Sistema para Análise Estatísticas e Genéticas), segundo EUCLYDES (1983), que utiliza o método de Gauss Newton puro.

De posse dos parâmetros da função difásica, calculou-se produção de leite total (PLT) por meio da integral da equação (1) para $\mathrm{t}=\mathrm{DL}$ (duração da lactação) como:

$\operatorname{PLT}_{\mathrm{DL}}=\sum_{\mathrm{i}=1}^{2}\left\{\mathrm{a}_{\mathrm{i}}\left[\tanh \left(\mathrm{b}_{\mathrm{i}}\left(\mathrm{DL}-\mathrm{c}_{\mathrm{i}}\right)\right)-\tanh \left(\mathrm{b}_{\mathrm{i}}\left(0-\mathrm{c}_{\mathrm{i}}\right)\right)\right]\right\}$,

em que: DL = duração da lactação; e $a_{i}, b_{i}, c_{i}$ e tanh = já definidos na equação (1).

A análise dos efeitos identificados, que poderiam contribuir para a variação da PLT e o cálculo da herdabilidade, foi realizada pelo método dos quadrados mínimos para números desiguais de informações nas subclasses - LSMLMW (Mixed Model LeastSquare and Maximum Likelihood Computer Program), segundo HARVEY (1990), utilizando-se o Modelo I: Modelo I:

$\mathrm{Y}_{\mathrm{j} \mathrm{k} \mathrm{m} m}=\mathrm{u}+\mathrm{P}_{\mathrm{i}}+\mathrm{B}_{\mathrm{ij}}+\mathrm{A}_{\mathrm{k}}+\mathrm{E}_{1}+(\mathrm{AE})_{\mathrm{kl}}+\mathrm{b}_{1}\left(\mathrm{I}_{\mathrm{j} \mathrm{jkm}}-\overline{\mathrm{I}}\right)+\mathrm{b}_{2}\left(\mathrm{I}_{\mathrm{ijk} \mathrm{k}}-\overline{\mathrm{I}}\right)^{2}+\mathrm{e}_{\mathrm{j} \mathrm{jkm}}$, em que $Y_{\mathrm{ijklm}}=$ produção de leite total avaliada na lactação $\mathrm{m}$, iniciada na estação 1 do ano $\mathrm{k}$, da cabra filha do reprodutor $\mathrm{j}$ e da fazenda-raça $\mathrm{i} ; \mathrm{u}=$ constante inerente aos dados; $\mathrm{P}_{\mathrm{i}}=$ efeito da fazenda-raça $\mathrm{i}$, $(\mathrm{i}=$ $1,2, \ldots, 16) ; \mathrm{B}_{\mathrm{ij}}=$ efeito do reprodutor $\mathrm{j}$, dentro de fazenda-raça i $(\mathrm{j}=1,2, . ., 95)$, suposto aleatório, em que $\operatorname{Bij} \sim \operatorname{NID}\left(0, \sigma_{B}^{2}\right) ; A_{k}=$ efeito de ano de parto $\mathrm{k}\left(\mathrm{k}=1986\right.$ a 1992); $\mathrm{E}_{1}=$ efeito da estação de parto 1 , sendo 1 = 1 fora da estação ( dez. - jun.), 2 início da estação (jul. - ago.) e 3 final da estação de nascimento (set. - nov.); (AE) ${ }_{\mathrm{kl}}$ = efeito da interação de ano de parto k e estação de parto $1 ; b_{1}$ e $b_{2}=$ coeficientes de regressão linear e quadrático da característica $\mathrm{I}_{\mathrm{ijklm}}$, em função da idade da cabra ao parto; $\mathrm{I}_{\mathrm{ijklm}}=$ idade da cabra ao parto, em meses, na lactação $\underline{m}$, iniciada na estação $\underline{1}$ do ano $\underline{\mathrm{k}}$, da cabra filha do reprodutor $\mathrm{j}$ e da fazenda-raça $\mathrm{i} ; \overline{\mathrm{I}}=$ média de idade das cabras ao parto; $\mathrm{e}_{\mathrm{ijklm}}=$ erro aleatório associado à informação $\mathrm{Y}_{\mathrm{ijklm}} \sim \operatorname{NID}\left(0, \boldsymbol{\sigma}_{\mathrm{W}}^{2}\right)$.

As estimativas de herdabilidade foram obtidas pela correlação intraclasse entre meias irmãs paternas (SILVA, 1980).

Para a comparação das médias de fazenda-raça, foi utilizada a análise de agrupamento (CRUZ e REGAZZI, 1994).

As estimativas de repetibilidade foram calculadas com base num conjunto de dados diferente daquele empregado para os cálculos de herdabilidade. Foram usadas 1222 observações de 404 cabras com pelo menos duas lactações.

A análise foi realizada pelo modelo $\mathrm{I}$, com a substituição do termo $\mathrm{B}_{\mathrm{ij}}$ por $\mathrm{C}_{\mathrm{ij}}$,

em que $\mathrm{Y}_{\mathrm{ijklm}}=$ característica avaliada na lactação $\underline{\mathrm{m}}$, iniciada na estação $\underline{1}$ do ano $\underline{\mathrm{k}}$, da cabra $\mathrm{j}$, dentro da classe fazenda-raça $\mathrm{i} ; \mathrm{C}_{\mathrm{ij}}=$ efeito da cabra $\mathrm{j}$ dentro da fazenda-raça $i$, sendo $j=1,2,3, \ldots, 11 \mathrm{e}$ $\mathrm{i}=1,2,3, \ldots 9$.

Os erros-padrão das estimativas de herdabilidade e repetibilidade foram calculados de acordo com SWIGER et al. (1964).

\section{Resultados e Discussão}

Na Figura 1 é apresentada a representação esquemática da função difásica para a lactação de uma cabra que produziu $907,74 \mathrm{~kg}$ de leite na lactação 


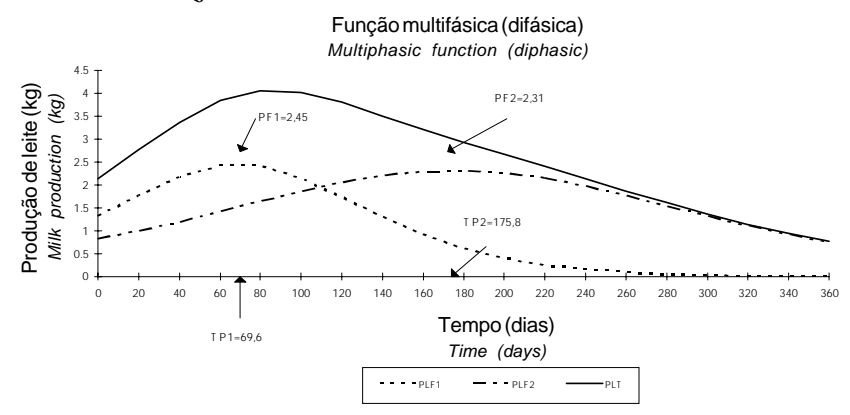

Figura 1 - Representação esquemática de uma lactação ajustada pela função multifásica (difásica).

Figure 1 - Eschematic representation of the lactation curve fitted by multiphasic function (Diphasic).

(343,05 kg na primeira e 564,69 kg na segunda fase), produção de leite no pico da fase 1 (PF1) de $2,45 \mathrm{~kg}$, e na fase 2 (PF2) de $2,31 \mathrm{~kg}$, tempo para atingir o pico na fase 1 (TP1) 69,6 dias e na fase 2 (TP2) 175,8 dias.

A média, o erro-padrão e o coeficiente de variação da PLT de 1336 lactações foram de $635,31 \pm 39,75 \mathrm{~kg}$ e $41,23 \%$, respectivamente.

O coeficiente de variação de $41,23 \%$ está situado entre os valores calculados a partir dos trabalhos de SINGH et al. (1970), BARHAT e CHOWDHARY (1978) e KANUJIA et al. (1988), que se encontram entre 26,05 e $41,65 \%$. O coeficiente de variação relativamente alto deste estudo possivelmente reflete condições desuniformes de alimentação, manejo e potencial genético dos animais nas diversas fazendas de criação.

A média de $635,31 \mathrm{~kg}$ de leite estimada neste trabalho foi maior que as encontradas em cabras nativas de regiões tropicais, como 146,70 e 131,70 kg para a raça Beetal na Índia, verificadas por SINGH et al. (1970) e PRAKASH et al. (1971), respectivamente; 201,96 kg para a Jamnapari na Índia, por SINGH et al. (1974); 102,96 kg para a média das raças Barbari, Jakharana, Marvari e Sirohi na Índia, por BARHAT e CHOWDHARY (1978); 229,00 kg para a Crioula no México, por MONTALDO et al. (1981); e 171,38 kg para a média de Beetal, Beetal x Black Bengal e Black Bengal x Beetal na Índia por KANUJIA et. al. (1988). Essas raças, de maneira geral, não são especializadas para a produção de leite e são criadas de maneira extensiva, recebendo em algumas épocas do ano, de acordo com a produção de leite, farelos de cereais.

O resultado obtido neste trabalho também foi superior àquele verificado em cabras especializadas exploradas em regiões tropicais, como para as raças Parda Alpina (310 kg) e Anglo Nubiana (289 kg), na Índia, criadas em regime de estabulação completa (GILL e DEV, 1972); mestiças de Crioulo com Parda Alpina, Granadina, Anglo Nubiano, Saanen e Toggenburg, com produções médias de 347,40; 227,11, 243,58; 329,73; e 326,77 kg, respectivamente, no México, mantidas em estabulação livre e alimentadas com feno de alfafa, silagem de sorgo e grãos de sorgo moídos (MONTALDO et al., 1981); cabras Pardas Alpinas no Egito, cuja média foi de 468,80 kg entre a primeira e a quinta ordem de parição, alimentadas com cevada, feno de leucena e forragens verdes (MOURAD, 1992); e de um rebanho de cabras Saanen no Brasil (RIBEIRO, 1997).

Com relação a raças especializadas, exploradas em regiões temperadas, o resultado deste estudo apresentou-se superior aos verificados na Noruega, com média de $576 \mathrm{~kg}$ (STEINE, 1975), e também superior ao das raças Saanen e Parda Alpina em primeira lactação, com médias de 512 e $456 \mathrm{~kg}$ na França (BOICHARD et al., 1989).

Entretanto, o resultado obtido foi inferior ao citado por KENNEDY et al. (1981) nos Estados Unidos para as raças Parda Alpina, Saanen e Toggenburg, com médias de 708, 710,5 e 716,5 kg, respectivamente; ALDERSON e POLLAK (1980) também nos Estados Unidos, com médias de 977,00; 960,00; 935,00; 925,00; e 769,00 kg para as raças Saanen, Parda Alpina, Toggenburg, LaMancha e Anglo Nubiana, respectivamente; e GIPSON e GROSSMAN (1989), que, usando a função difásica para o ajustamento das lactações, relataram médias de 872,80; 753,20; 704,63; 899,90 ; e $868,33 \mathrm{~kg}$ para as raças Parda Alpina, LaMancha, Anglo Nubiana, Saanen e Toggenburg, respectivamente, em primeira parição.

Com a comparação dos resultados do presente estudo, verifica-se que o nível de produção das cabras exploradas na região Sudeste do Brasil se encontra em um nível médio de produção. A diferença observada em relação às cabras exploradas nos Estados Unidos possivelmente deve-se à falta de um programa efetivo de seleção dos animais, além de deficiências no manejo, clima e na alimentação.

O resumo da análise de variância da produção de leite encontra-se na Tabela 1.

Ainteração anox estaçãodo partoinfluenciou de modo significativo $(\mathrm{P}<0,01)$ a produção de leite (Tabela 1$)$, sendo responsável por 2,25\% da variação total ocorrida. Esta interação deve-se às diferenças observadas entre as estações nos anos de 1987 a 1992 (Tabela 2). 
Tabela 1 - Resumo da análise de variância da produção de leite total Table 1 - Summary of variance analysis for total milk production

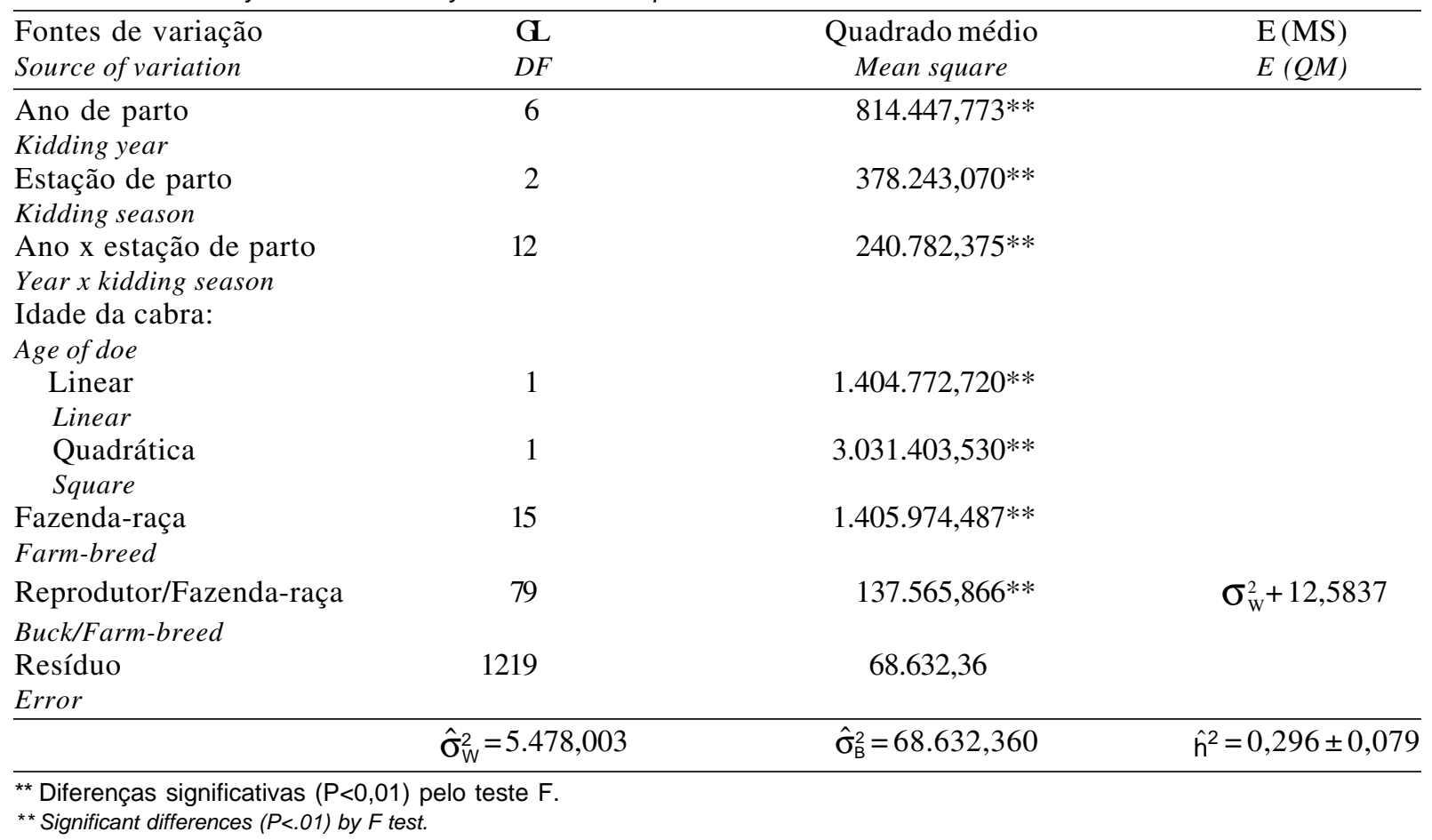

Observa-se que a PLT no ano de 1987 foi maior na estação 2 que na estação 1 , e estas não diferiram da estação 3. No ano de 1992, a maior produção foi observada na estação 1 , seguida das estações 2 e 3 .

No ano de 1992, a maior PLT observada na estação 1 pode ser atribuída ao maior tempo de pico da fase 1 e menor produção de leite inicial da fase 1 observada na estação 1 e à duração da fase $2 \mathrm{e}$ produção de leite da fase 2 maiores na estação 1 que na 3. Além de a duração da lactação nos anos de 1986 a 1989 e 1992 ter se apresentado na estação 3 com médias menores ou, no máximo, iguais às outras duas e do maior intervalo de partos observado no ano de 1991 na estação 1, que certamente possibilitou melhores condições de recuperação para as cabras paridas nessa estação no ano de 1992 (GONÇALVES, 1996).

Somam-se a isso os seguintes fatos:

1. Para as cabras paridas na estação 3, o final da lactação coincide com a época do ano em que a quantidade equalidade do alimento, principalmente o volumoso, diminuem, contribuindo para reduzir a produção de leite.

2. As lactações iniciadas na estação 3 (set.-nov.) apresentaram-se mais curtas que as demais, possivelmente em virtude da proximidade da estação de monta, que ocorre no início do ano, levando as cabras a um menor período de serviço e intervalo de partos (IDP), conforme publicado por GONÇALVES et al. (1997). Em decorrência disso, houve aumento no tempo em que as cabras permaneciam em gestação e lactação simultaneamente, colaborando também para a redução da PLT, possivelmente em virtude do deslocamento de nutrientes para o feto em formação.

3. O início da lactação, em que ocorrem as maiores necessidades nutricionais, coincide com o princípio das chuvas da primavera e a elevação da temperatura, que pode comprometer a ingestão de alimentos, como apontado por QUITTET (1978) e APPLEMAN e DELOUCHE (1958).

Na Figura 2 encontra-se representada, de forma quadrática, a evolução da produção de leite total, em função do ano de parição das cabras e as equações de regressão para cada estação de nascimento. $\mathrm{Na}$ Tabela 3 são apresentados os coeficientes de regressão, o ponto de máximo, o ano de ocorrência e o coeficiente de determinação para cada estação.

Observa-se, na Figura 2, que, nas três estações, inicialmente houve aumento da produção de leite de 1986 a 1989, que permaneceu em um patamar elevado até 1990 , seguido posteriormente de ligeira queda em 1991 e 1992.

Efeito significativo da interação ano x estação de parto sobre a produção de leite foi observado por MAVROGENIS et al. (1984) na produção de leite 


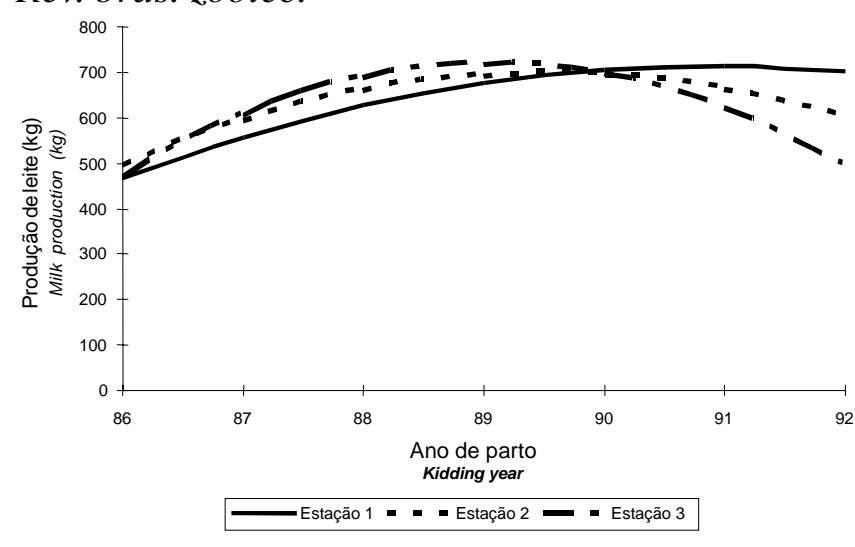

Figura 2 - Regressão da produção de leite total (PLT), para cada estação, em função do ano de parto das cabras.

Figure 2 - Regression of total milk production, within season on kidding years.

avaliada no período de 90 a 150 dias após o desmame de cabras da raça Damascus no Chipre.

A maior produção de leite total observada de 1988 a 1990 possivelmente deveu-se à maior produção de leite total constatada na estação de nascimento 3(set.nov.) nesses anos, o que colaborou para aumentar a produção de leite total nesse período (Tabela 2). Este resultado difere dos obtidos por SINGH et al. (1970),
PRAKASH et al. (1971) e BARHAT e CHOWDHARY (1978), que observaram apenas efeito de ano na produção de leite, porém sem avaliar a interação de ano com outros efeitos. Esses autores não constataram tendência definida da produção de leite em relação aos anos de parto.

O resultado obtido neste trabalho para o ano de 1987, em que se observa efeito significativo da estação de parto na produção de leite, sendo a maior produção observada no início da estação (estação 1), coincide com a primavera, que está de acordo com os encontrados tanto em países tropicais, como a Índia (SINGHet al., 1970; PRAKASH et al., 1971) e o México (MONTALDO et al., 1981), quanto em países temperados, como Noruega (STEINE, 1975), Estados Unidos (ALDERSON e POLLAK 1980), Chipre (MAVROGENIS et al., 1984) e Egito (MOURAD, 1992).

A causa de variação fazenda-raça influenciou significativamente $(\mathrm{P}<0,01)$ a produção de leite (Tabela 1) e foi responsável por 16,04\% de variação total da produção de leite. As estimativas das médias ajustadas para as produções de leite, segundo a fazenda-raça, estão na Tabela 4 e na Figura 3.

Tabela 2 - Número de observações, médias ajustadas e respectivos erros-padrão da produção de leite, segundo 0 ano e a estação de parto

Table 2 - Number of observations, least square means and standard error for total milk production, according to year $x$ kidding season

\begin{tabular}{|c|c|c|c|}
\hline \multirow[t]{2}{*}{$\begin{array}{l}\text { Ano } \\
\text { Kidding year }\end{array}$} & \multicolumn{3}{|c|}{$\begin{array}{c}\text { Estação } \\
\text { Kidding season }\end{array}$} \\
\hline & $\begin{array}{l}1 \text { (Dez.-Jun.) } \\
1 \text { (Dec.-Jun.) }\end{array}$ & $\begin{array}{c}\text { (Jul.-Ago.) } \\
2 \text { (Jul.-Aug.) }\end{array}$ & $\begin{array}{l}3 \text { (Set.-Nov) } \\
3 \text { (Sep.-Nov.) }\end{array}$ \\
\hline 1986 & $\begin{array}{c}463,72 \pm 76,12^{a} \\
(22)\end{array}$ & $\begin{array}{c}489,13 \pm 62,76^{a} \\
(49)\end{array}$ & $\begin{array}{c}423,60 \pm 70,35^{\mathrm{a}} \\
(63)\end{array}$ \\
\hline 1987 & $\begin{array}{c}532,36 \pm 49,07^{b} \\
(163)\end{array}$ & $\begin{array}{c}657,84 \pm 56,69^{a} \\
(49)\end{array}$ & $\begin{array}{c}575,14 \pm 53,03^{\mathrm{ab}} \\
(80)\end{array}$ \\
\hline 1988 & $\begin{array}{c}758,64 \pm 51,86^{a} \\
(72)\end{array}$ & $\begin{array}{c}726,03 \pm 53,73^{\mathrm{a}} \\
(61)\end{array}$ & $\begin{array}{c}765,38 \pm 52,77^{b} \\
(67)\end{array}$ \\
\hline 1989 & $\begin{array}{c}782,81 \pm 53,05^{\mathrm{a}} \\
(60)\end{array}$ & $\begin{array}{c}714,28 \pm 48,03^{\mathrm{a}} \\
(102)\end{array}$ & $\begin{array}{c}653,98 \pm 61,67^{a} \\
(36)\end{array}$ \\
\hline 1990 & $\begin{array}{c}620,87 \pm 98,36^{\mathrm{a}} \\
(9)\end{array}$ & $\begin{array}{c}664,80 \pm 51,46^{\mathrm{a}} \\
(74)\end{array}$ & $\begin{array}{c}650,22 \pm 51,75^{a} \\
(76)\end{array}$ \\
\hline 1991 & $\begin{array}{c}625,08 \pm 55,12^{a} \\
(63)\end{array}$ & $\begin{array}{c}704,61 \pm 53,58^{a} \\
(79)\end{array}$ & $\begin{array}{c}555,97 \pm 71,95^{\mathrm{a}} \\
(23)\end{array}$ \\
\hline 1992 & $\begin{array}{c}818,13 \pm 63,02^{\mathrm{a}} \\
(41)\end{array}$ & $\begin{array}{c}649,14 \pm 58,01 \mathrm{~b} \\
(74)\end{array}$ & $\begin{array}{c}509,71 \pm 58,41^{\mathrm{c}} \\
(73)\end{array}$ \\
\hline Geral & $657,37 \pm 43,46$ & $657,98 \pm 40,91$ & $590,57 \pm 41,59$ \\
\hline Overall & $(430)$ & (488) & (418) \\
\hline
\end{tabular}

Dentro de cada ano, médias seguidas de pelo menos uma mesma letra não diferem pelo teste Tukey $(\mathrm{P}>0,01)$.

Means within years followed by at least one letter do not differ $(P>$.01) accoding to Tukey test. 
Tabela 3 - Coeficientes de regressão, ponto de máximo, ano de máxima observação e coeficiente de determinação para a PLT em função do ano de parto para cada estação

Table 3 - Coefficients of regression, maximum yield, year of maximum yield and coefficient of determination $\left(R^{2}\right)$ for total milk production, according to year of birth within season

\begin{tabular}{|c|c|c|c|c|c|c|}
\hline \multirow[b]{2}{*}{$\begin{array}{c}\text { Estação } \\
\text { Kidding season }\end{array}$} & \multicolumn{3}{|c|}{$\begin{array}{c}\text { Coeficientes } \\
\text { Coefficients }\end{array}$} & \multirow{2}{*}{$\begin{array}{l}\text { Ponto de } \\
\text { máximo } \\
\text { Maximum } \\
\quad \text { yield }\end{array}$} & \multirow{2}{*}{$\begin{array}{c}\text { Ano de máx. } \\
\text { obs. } \\
\text { Year of } \\
\text { maximum yield }\end{array}$} & \multirow{2}{*}{$\begin{array}{l}\mathrm{R}^{2} \\
R^{2}\end{array}$} \\
\hline & $\begin{array}{c}\text { Constante } \\
\text { Constant }\end{array}$ & $\begin{array}{l}\text { Linear } \\
\text { Linear }\end{array}$ & $\begin{array}{c}\text { Quadrático } \\
\text { Quadratic }\end{array}$ & & & \\
\hline 1 (Dez.-Jun.) & $-84000,60$ & 1863,68 & $-10,25$ & 737,26 & 90,94 & 0,4927 \\
\hline $\begin{array}{l}1 \text { (Dec.-Jun.) } \\
2 \text { (Jul.-Ago.) } \\
2 \text { (Jul.-Aug.) }\end{array}$ & $-127137,00$ & 2854,94 & $-15,94$ & 728,07 & 89,57 & 0,7952 \\
\hline $\begin{array}{l}3 \text { (Set.-Nov.) } \\
3 \text { (Sep.-Nov.) }\end{array}$ & $-206788,00$ & 4658,80 & $-26,15$ & 695,34 & 89,07 & 0,7815 \\
\hline
\end{tabular}

As comparações entre os níveis da fazenda-raça, realizadas por meio da análise de agrupamento, constam da Tabela 4. As médias foram reunidas em quatro grupos. Em virtude da complexidade de estabelecer uma relação biologicamente válida para todos os níveis da fazenda-raça, optou-se inicialmente pela discussão dos níveis da raça dentro de uma fazenda e, após, dos níveis da fazenda dentro de determinada raça.

Comparando-se as raças dentro de determinada fazenda, constatou-se que na fazenda 4 a raça Saanen apresentou produção de leite maior que a raça Parda Alpina. Na fazenda 3, a situação inverteu-se, sendo a Parda Alpina superior à Saanen. Na fazenda 6 a Saanen foi superior à Toggenburg e na fazenda 2 a

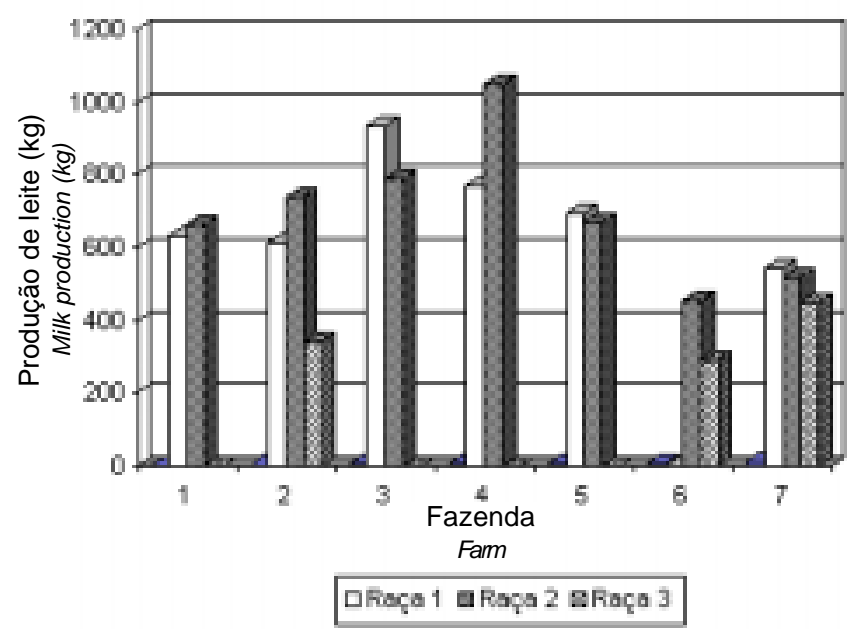

Figura 3 - Produção de leite total, segundo a combinação fazenda-raça.

Figure 3 - Total milk production, according to farm-breed combination.
Saanen e a Parda Alpina foram superiores à Toggenburg. Com esses resultados verifica-se que a Toggenburg foi inferior às outras duas.

A comparação da raça Parda Alpina em várias fazendas revelou que ocorreu maior produção na fazenda 3 que nas 1, 2, 4 e 5, que não diferiram e, por sua vez, tiveram produção maior que na fazenda 7 . A Saanen teve a maior média na fazenda 4 . As médias nas fazendas 1, 2, 3 e 5 não diferiram entre si e foram maiores que nas fazendas 6 e 7. A Toggenburg obteve a maior média na fazenda 7 , e as médias das fazendas 2 e 6 foram semelhantes.

Pelo comportamento diferenciado das raças Parda Alpina e Saanen nas diversas fazendas, pode-se verificar que ou elas respondem a melhorias nas condições de manejo nas várias fazendas, ou existe diferença no potencial genético destas raças nas várias fazendas, ou ambas. Este comportamento não ocorreu com a Toggenburg, possivelmente em virtude do baixo potencial de produção desta raça, ou seja, o ambiente de criação não estabeleceu limites à sua capacidade de produção.

A superioridade da raça Saanen sobre a Parda Alpina e desta sobre a Toggenburg com relação à PLT foi observada por ALDERSON e POLLAK (1980) nos Estados Unidos

Efeito significativo de raça foi verificado por BARHAT e CHOWDHARY (1978) e KANUJIA et al. (1988) para raças nativas da Índia, que tiveram médias de produção de leite bastante inferiores ao deste estudo, situando-se na faixa de 100 a $200 \mathrm{~kg}$.

Já os mestiços de raças nativas com raças exóticas tiveram desempenho superior aos crioulos, como observado por CHAWLA e BHATNAGAR (1984) e MONTALDO et al. (1981), em que a produção de leite se encontra entre 200 e $350 \mathrm{~kg}$ de leite. 
Rev. bras. zootec.

Tabela 4 - Número de observações, médias ajustadas e respectivos erros-padrão da produção de leite, segundo a combinação fazenda-raça

Table 4 - Number of observations, least square means and standard errors of total milk production, according to farm-breed combination

\begin{tabular}{lccc}
\hline $\begin{array}{l}\text { Fazenda } \\
\text { Farm }\end{array}$ & $\begin{array}{c}\text { Raça } \\
\text { Breed }\end{array}$ & $\begin{array}{c}\text { Número de observações } \\
\text { Number of observations }\end{array}$ & $\begin{array}{c}\text { Médias ajustadas }(\mathrm{kg}) * \\
\text { Least square means }(\mathrm{kg})\end{array}$ \\
\hline 1 & 1 & 8 & $630,822 \pm 115,456^{\mathrm{b}}$ \\
2 & 57 & $661,281 \pm 49,556^{\mathrm{b}}$ \\
& 2 & 9 & $613,824 \pm 98,517^{\mathrm{b}}$ \\
3 & 1 & 11 & $741,878 \pm 87,971^{\mathrm{b}}$ \\
& 2 & 4 & $351,685 \pm 142,079^{\mathrm{b}}$ \\
4 & 3 & 23 & $935,213 \pm 80,649^{\mathrm{d}}$ \\
5 & 1 & 30 & $787,194 \pm 76,129^{\mathrm{b}}$ \\
& 2 & 58 & $770,464 \pm 81,592^{\mathrm{b}}$ \\
6 & 1 & 110 & $1048,119 \pm 50,819^{\mathrm{d}}$ \\
& 1 & 298 & $695,198 \pm 47,121^{\mathrm{b}}$ \\
7 & 2 & 7 & $670,230 \pm 30,640^{\mathrm{b}}$ \\
& 2 & 13 & $454,540 \pm 125,264^{\mathrm{a}}$ \\
& 3 & 469 & $290,405 \pm 93,505^{\mathrm{c}}$ \\
Geral & 1 & 60 & $546,384 \pm 41,165^{\mathrm{a}}$ \\
Overall & 2 & 159 & $517,574 \pm 54,165^{\mathrm{a}}$ \\
\hline
\end{tabular}

Médias com letras iguais para o efeito da fazenda-raça foram classificadas como pertencentes a um mesmo grupo pela análise de agrupamento, utilizando-se o método de Tocher $(q=112,91)$.

Means the same letter within the same farm-breed combination were clustered together, according to Tocher method ( $q=112.91)$.

A produção de leite das raças especializadas foi superior à dos nativos e dos mestiços, quer em condições de clima tropical ou temperado. Os valores deste estudo foram maiores que os relatados por GILL e DEV (1972), na Índia, e menores que os de ALDERSON e POLLAK (1980) e GIPSON e GROSSMAN (1989), nos Estados Unidos.

Levando-se em consideração as médias de produção evidenciadas neste estudo (Tabela 4) e as condições de criação, parece indicada a continuidade da exploração das raças Saanen e Parda Alpina, que podem não ter apresentado melhores resultados em virtude da inexistência de um programa de seleção e melhoramento dos animais. Além disso, merece consideração o fato evidenciado neste estudo de possível interação genótipo $\mathrm{x}$ ambiente, representada pelas diferenças de desempenho das raças nas diferentes fazendas (Figura 3), que também foi apontado por MONTALDO et al.(1981) e BOICHARD et al. (1989).

A idade da cabra ao parto influenciou $(\mathrm{P}<0,01)$ a produção de leite de forma quadrática (Tabela 1). A equação e a respectiva curva de regressão da produção de leite, em função da idade ao parto, encontram-se representadas na Figura 4. A idade de máxima produção correspondeu a 46,66 meses.

Considerando a idade ao primeiro parto de 607,18 dias e o intervalo de partos de 339,98 encontrado nesse estudo, a idade de 46,66 meses está entre o terceiro (42,90 meses) e o quarto (54,23 meses) partos. As cabras nos dois primeiros ciclos (gestação-lactação) reprodutivos ainda estão em crescimento (FEHR, 1981) e parte dos nutrientes provenientes da alimentação é destinada ao desenvolvimento dos diversos tecidos corporais, inclusive o tecido secretor da glândula mamaria em detrimento da produção de leite (PRAKASH et al., 1971).

Estes resultados concordam com os obtidos na Índia para a produção de leite nas raças nativas de países tropicais por PRAKASH et al. (1971), SINGH e SINGH (1974) e CHAWLA e BHATNAGAR (1984), que relataram a máxima produção na terceira, segunda e terceira lactações, respectivamente, ou seja, entre três e quatro anos de idade. Foram também semelhantes aos obtidos para as raças especializadas estudadas por RONNINGEN (1965), STEINE (1975), MONTALDO et al. (1981) e SUBIRES et al. (1988), que constataram a máxima produção entre três e sete e terceira e quarta lactações. Porém, foram superiores à idade relatada por ALDERSON e POLLAK (1980), em que a máxima produção ocorreu entre 31 e 39 meses, terceira lactação. 


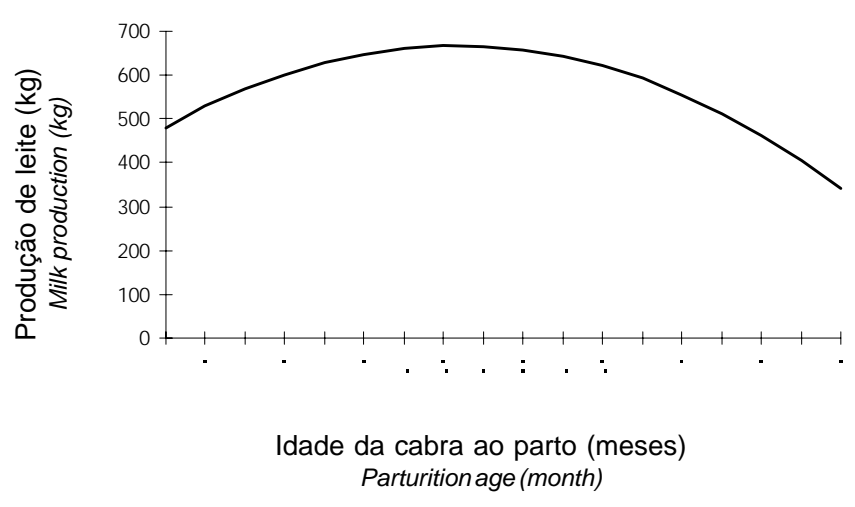

Figura 4 - Regressão da produção de leite total na lactação, em função da idade da cabra ao parto.

Figure 4 - Regression of total milk production on goat age at parturition.

\section{Herdabilidade}

A estimativa de herdabilidade da produção de leite total, 0,296 $\pm 0,079$ (Tabela 1), assemelhou-se mais às encontradas em raças nativas em regiões tropicais, cujos valores estão entre 0,15 e 0,35 , com média de 0,28 (SINGH et al., 1970; PRAKASH et al., 1971; CONSTANTINOU et al., 1985; MAVROGENIS et al., 1984), que as obtidas em raças especializadas em países de clima temperado, cujos valores estão entre 0,28 e 0,72 e média de 0,47 (RONNINGEN, 1965; RONNINGEN, 1967; STEINE, 1976; ILOEJE et al., 1981; KENNEDY et al., 1982; SULLIVAN et al., 1986; BOICHARD et al., 1989).

A estimativa de herdabilidade deste trabalho foi superior a 0,09 relatada por RIBEIRO (1997), no Brasil, que considerou este baixo valor, devido ao pequeno número de observações.

Porém, como exposto por FALCONER (1960), esta grande variação nas estimativas de herdabilidade é previsível, porque é uma estatística da relação entre duas variâncias, sendo característica de uma dada população em determinado meio num tempo definido.

Sendo o valor da estimativa de herdabilidade da produção de leite 0,296 , e por ser esta característica limitada ao sexo, sugerem-se o teste de progênie como método de seleção mais eficiente para a seleção dos bodes e o uso da inseminação artificial como processo de disseminação do material genético selecionado. Outra razão para o uso do teste de progênie e inseminação artificial é o pequeno intervalo de geração dos caprinos, comparado ao dos bovinos, que possibilita a obtenção de maiores ganhos genéticos anuais.

\section{Repetibilidade}

A estimativa de repetibilidade para produção de leite, $0,277 \pm 0,033$, obtida neste estudo, foi relativamente baixa, levando à conclusão de que uma única observação no animal não pode representar sua real capacidade de produção. Portanto, seriam necessárias mais observações do indivíduo para a obtenção de maior precisão, porém isso provocaria aumento no intervalo de gerações e, como conseqüência, diminuição do progresso genético.

Este valor assemelha-se a 0,20, obtido por RIBEIRO (1997), que também atribuiu seu baixo valor ao número limitado de observações.

Os valores da repetibilidade para a produção de leite relatados na literatura para cabras especializadas em regiões de clima temperado encontram-se entre $0,38 \mathrm{e} 0,63$ (RONNINGEN, 1965; RONNINGEN, 1967; STEINE, 1976; KENNEDY et al., 1981; GROSSMAN et al., 1986), e para cabras especializadas cruzadas com Crioulo em vários graus de sangue, no México (clima tropical), MONTALDO et al. (1982) encontraram valor de 0,59. Já para as raças nativas na Índia; o dado deste estudo é superior a 0,18, relatado por KUMAR et al. (1962).

O valor de herdabilidade superior ao de repetibilidade, estimado neste trabalho, deve-se, possivelmente, ao fato de as estimativas serem originadas de conjuntos de dados diferentes, em decorrência da disponibilidade de informações.

\section{Conclusões}

As maiores produções de leite totais foram observadas nas estações 1 (Dez. - Jun.) e 2 (Jul.-Ago.), em decorrência da maior duração da lactação, maiores intervalos de partos e qualidade e quantidade do alimento volumoso.

Ocorreu alternância de superioridade da PLT das raças Parda Alpina e Saanen dentro de determinada fazenda, porém a raça Toggenburg sempre apresentou média inferior às outras duas.

A idade de máxima produção de leite ocorreu aos 46,66 meses ou entre o terceiro e quarto partos, considerando-se a idade ao primeiro parto e o intervalo de parto dos animais em estudo.

Os valores de herdabilidade e repetibilidade da PLT dos caprinos sugerem o uso de teste de progênie para a seleção dos bodes. 


\section{Referências Bibliográficas}

ALDERSON, A., POLLAK, E.J. 1980. Age-season adjustment factors for milk and fat of dairy goats. J. Dairy Sci., 63:148-151.

APPLEMAN, R.D., DELOUCHE, J.C. 1958. Behavioral, physiological and biochemical responses of goats to temperature 0 to $40^{\circ} \mathrm{C}$. J. Anim. Sci., 17:326-335.

BARHAT, N.K., CHOWDHARY, M.S. 1978. Factors affecting some production traits in Rajasthani goats. Ind. J. Dairy Sci., 31:185-188.

BOICHARD, D., BOULOC, N., RICORDEAU, G. et al. 1989. Genetic parameters for first lactation dairy traits in the Alpine and Saanen goat breeds. Genet. Sel. Evol., 21:205-215.

BOUILLON, J., RICORDEAU, G. 1976. Genetic parameters of body weight and milk performance for goat in progenytesting station. Estimation of direct and indirect responses to selection. Annales de Génétique et de Sélection Animale, $8: 109$.

CHAWLA, D.S., BHATNAGAR, D.S. 1984. First 150; day lactation yield in different crossbred goats. Ind. J. Anim. Sci, 54:822-824.

CONSTANTINOU, A., BEUING, R., MAVROGENIS, A.P. 1985. Genetic and phenotypic parameters for some reproductive and milk production characters of the Damascus goat. Z. Tierz. Züechtungsbiol., 102:301-307.

CRUZ, C.D., REGAZZI, A. 1994. Modelos biométricos aplicados ao melhoramento genético. Viçosa, MG: UFV. 390p.

EUCLYDES, R.F. 1983. Manual de utilização do sistema SAEG (Sistema para Análises Estatísticas e Genéticas). Viçosa: UFV. 59p.

FALCONER, D.S. 1960. Introduction to quantitative genetics. 1.ed. Edinburg: Ronald Press. 365p.

FEHR, P.M. 1981. Growth. In: GALL, C. (Ed.) Goat production. 1.ed, New York: Academic Press. p.253-283.

FRESNO, M., RODERO, J.M., SERRANO, I. et al. A. 1991. Evolución de la producción de leche de la población caprina tinerfeña según algunos factores ambientales. Av. Aliment. Mejora Anim., 31:265-267.

GALL, C. 1981. Milk production. In: GALL, C. (Ed.) Goat production. 1.ed, New York: Academic Press. p.309-344.

GILL, G.S., DEV, D.S. 1972. Performance of two exotic breeds of goats under Indian conditions. Ind. J. Anim. Prod., 3:173-178.

GIPSON, T.A., GROSSMAN, M. 1989. Diphasic analysis of lactation curves in dairy goats. J. Dairy Sci., 72:1035-1044.

GONÇALVES, H.C. Fatores genéticos e de meio em algumas características produtivas e reprodutivas de caprinos. Viçosa, MG: UFV, 1996. 141p. Tese (Doutorado em Zootecnia) - Universidade Federal de Viçosa, 1996.

GONÇALVES, H.C., SILVA, M.A., RAMOS, A.A. et al. 1997. Fatores genéticos e de meio no intervalo de partos de caprinos leiteiros. R. Bras. Zootec., 26(5):905-913.

GROSSMAN, M., FERNANDO, R.L., MOHAMMAD, W.A. et al. 1986. Correlations between parities for lactation traits in United State dairy goats. J. Dairy Sci., 69:1917-1921.

GROSSMAN, M., KOOPS, W.J. 1988. Multiphasic analysis of lactation curves in dairy cattle. J. Dairy Sci., 71:1598-1608.

GROSSMAN, M., KOOPS, W.J. 1988a. Multiphasic analysis of growth curves in chickens. Poult. Sci., 67:33-42.

HARVEY, W.R. 1990. User's guide for LSMLMW and (Mixed model least-squares and maximum likelihood computer program), wooster. Ohio: Ohio State University. 91p.

ILOEJE, M.U., VAN VLECK, L.D., WIGGANS, G.R. 1981. Components of variance for milk and fat yields in dairy goats. J. Dairy Sci., 64:2290-2293.
KANUJIA, A.S., PANDER, B.L., SHARMA, J.S. 1988. First lactation milk production potential in Beetal and its crosses with Black Bengal. Ind. J. Dairy Sci., 41:351-352.

KENNEDY, B.W., FINLEY, C.M., BRADFORD, G.E. 1982. Phenotypic and genetic relationships between reproduction and milk production in goats. J. Dairy Sci., 65:2373-2383.

KENNEDY, B.W., FINLEY, C.M., POLLAK, E.J. et al. 1981. Joint effects of parity, age, and season of kidding on milk and fat yields in dairy goats. J. Dairy Sci., 64:1707-1712.

KUMAR, S., SINGH, G., KUMAR, A.N. 1962. Repeatability estimates of some economic characters in the Beetal goat. Ind. J. Dairy Sci., 15:101-104.

MAVROGENIS, A.P., CONSTANTINOU, A., LOUCA, A. 1984. Environmental and genetic causes of variation in production traits of Damascus goats. Anim. Prod., 33:99-104.

MONTALDO, H., TAPIA, G., JUÁREZ, A. 1981. Algumos factores geneticos y ambientales que influyen sobre la produccion de leche y el intervalo entre partos en cabras. Tec. Pecu. Mex., 41:32-44.

MONTALDO, H., ROSALES, J., JÚAREZ, A. 1982. Coeficients de repetibilidad para algunas caracteristicas de produccion de leche y reproducion en cabras. Tec. Pecu. Mex., 43:70-72.

MOURAD, M. 1992. Effects of month of kidding, parity and litter size on milk yield of Alpine goats in Egypt. Small Ruminant Res., 8:41-46.

PRAKASH, C., ACHARYA, R.M., DHILLON, J.S. 1971. Sources of variation in milk production in Beetal goats. Indian J.Anim. Sci., 41:356-360.

QUITTET, E. 1978. La cabra. Guia practica para el ganadero. 1.ed. Madrid: Mundi-Prensa. 321p.

RIBEIRO, A.C. Estudo dos efeitos genéticos e de ambiente sobre características de importância econômica em caprinos da raça Saanen. Jaboticabal, SP: FCAV, 1997. 119p. Dissertação (Mestrado em Zootecnia) - Faculdade de Ciências Agrárias e Veterinárias/ Universidade Estadual Paulista, 1997.

RONNINGEN, K. 1965. Causes of variation in the flavor intensity of goat milk. Acta Agric. Scand., 15:301-342.

RONNINGEN, K. 1967. A study of genetic parameters for milk characteristics in goat. Meld. Nor. Landbrukshogsk., 46:1-17.

SILVA, M. A. 1980. Melhoramento animal, métodos de estimação de componentes genéticos. Viçosa, MG: UFV. 49p.

SINGH, R.N., ACHARYA, R.M., BISWAS, D.K. 1970. Evaluation of genetic and non-genetic factors affecting some economic traits in goats. Acta Agric. Scand., 20:10-14.

SINGH, B. B., SINGH, B.P. 1974. Performances of Jamnapari goats. Ind. Vet. J., 51:326-332.

STEINE, T.A. 1975. Factors affecting traits of economic importance in goat. Meld. Nor. Landbrukshogsk., 54:1-30.

STEINE, T.A. 1976. Genetic and phenotypic parameters for production traits in goat. Meld. Nor. Landbrukshogsk., 55:1-19.

SUBIRES, J., LARA, L., FERRANDO, G. et al. 1988. Factores que condicionan la productividad lechera de la cabra. Número de lactación y tipo de parto. Arch. Zootec., 37:145-153.

SULLIVAN, B.P., KENNEDY, B.W., SCHAEFFER, L.R. 1986. Heritabilities, repetabilities and correlations for milk, fat, and protein yields in goats. J. Dairy Sci., 69:100. (Abstracts).

SWIGER, L.A., HARVEY, W.R., EVERSON, D.O. et al. 1964. The variance of intra-class correlation involving groups with one observation. Biometrics, 20:818-826.

Recebido em: 14/09/99

Aceito em: 13/12/00 